\title{
Digital Ischemia in Patients with Solid Tumors: A Case Report and Review of the Literature
}

\author{
Lucia Raimondo, Chiara Alessandra Cella, Roberto Moretto, Elide Matano, Chiara Carlomagno
}

Dipartimento di Endocrinologia ed Oncologia Molecolare e Clinica, Università Federico II, Naples, Italy. Email: chiara.carlomagno@unina.it

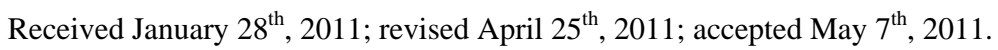

\begin{abstract}
Digital ischemia is a rare paraneoplastic phenomenon associated with various malignancies, especially adenocarcinomas. We reported a case of digital ischemia onset during treatment with capecitabine + oxaliplatin, letrozole and epoetin-beta in a 75-year old woman with colon and breast cancer and a secondary hepatic lesion. A literature review disclosed 68 cases of solid neoplasms associated with digital ischemia. The proposed mechanisms of such clinical manifestations are various: vasospasm due to sympathetic hyperactivity, arteritis induced by tumour antigen-antibody complexes deposition or as consequence of immune deregulation, blood hyperviscosity, hypercoagulability or peripheral thrombosis. In the present case, clinical and laboratory evaluations failed to reveal evidence of thrombosis, arteritis, inherited or acquired hypercoagulable state and we postulated the peripheral vasospasm and the sympathetic activity (possibly due to chemotherapy drugs) as potential causes of the digital ischemia.
\end{abstract}

Keywords: Breast Cancer, Colon Cancer, Digital Ischemia, Paraneoplastic Syndrome

\section{Introduction}

Digital ischemia is a rare, paraneoplastic phenomenon associated with various malignant tumours, especially adenocarcinomas which cause has not been completely clarified [1].

The earliest paraneoplastic syndrome involving the vascular system was described by Trousseau in 1865 when he noticed that some patients with unexpected, unusual, or migratory thromboses later manifested a visceral malignancy. This clinical manifestation was frequently associated to chronic disseminated intravascular coagulopathy, platelet-rich microthrombi, microangiopathic hemolytic anemia, verrucous endocarditis, and thromboembolic problems. Nowadays, Trousseau's syndrome is defined as a situation in which thrombotic problems arisen in patients with either an occult or a recently diagnosed carcinoma cannot be explained by any other obvious factors [2].

Treatments (chemotherapy, endocrine therapy or supportive drugs) cannot be excluded as possible cause of digital ischemia.

\section{Case Report}

A 75-year old non-smoker woman, HCV+, with a history of hypertension, diabetes, and an episode of arterial thrombosis of inferior right limb, at the end of 2009 received the diagnosis of colon cancer, breast cancer and one secondary hepatic lesion. She had underwent radical mastectomy plus axillary lymph-node dissection and histology was: ductal infiltrating carcinoma, TNM stage pT2G2N0, Erb2 neg, ER+++, Ki67 = 15\%. Due to persistent elevation of CEA serum level $(17.4 \mathrm{ng} / \mathrm{ml}$ pre-mastectomy; $107 \mathrm{ng} / \mathrm{ml}$ post-mastectomy) she made colonoscopy and total body CT scan revealing a colon cancer plus one hepatic secondary lesion. She underwent a right emicolectomy; histology accounted for adenocarcinoma, TNM stage pT3G1N1. During surgery, it was impossible to make a biopsy to characterize the hepatic lesion.

On February 2010 she was hospitalized in emergency because of abdominal pain. An abdominal magnetic resonance imaging showed a picture suggesting pancreatitis (pancreatic increased volume with oedema, and signs of inflammation in the distal tract of the bile duct) and the presence of one nodule ( $\varnothing 37 \mathrm{~mm}$ ) between VII and VIII hepatic segment and a small $(\varnothing<1 \mathrm{~cm})$ nodular lesion at VIII hepatic segment.

On March 2010 the patient referred to our Institution. As it was unfeasible a cytological or histological diagnosis of the liver nodules, due to the more advanced TNM 
stage of the colon primary tumor, the high CEA serum level and being the liver the most frequent site of metastasis for colon cancer, we trusted the liver nodules to be metastases from colon cancer. Physical examination was unremarkable; WHO performance status $=0$. Investigations: CEA $14.9 \mathrm{ng} / \mathrm{ml}$, liver and kidney function within normal values, Quick, Aptt, and blood count within normal ranges with the exception of haemoglobin $9.5 \mathrm{~g} / \mathrm{dl}$; cardiac ultrasonography was normal.

The patient started chemotherapy with XELOX regimen (Oxaliplatin $130 \mathrm{mg} / \mathrm{m}^{2}$ i.v. day 1 , capecitabine $1000 \mathrm{mg} / \mathrm{m}^{2} /$ bid orally days 1 - 14, every 21 days) plus adjuvant hormonal treatment with letrozole $2.5 \mathrm{mg} /$ day. Supportive and concomitant therapies were: trivalent iron $6.25 \mathrm{mg} /$ day, metphormine $400 \mathrm{mg} / \mathrm{bid}$, lisinopril 10 $\mathrm{mg} /$ day, aspirin $100 \mathrm{mg} /$ day.

Chemotherapy was not well tolerated. During the first cycle, the patient experienced G1 diarrhoea and the persistence of G2 anaemia, thus the dosage of the second cycle was reduced to $75 \%$ and epoetin-beta was added. Toxicity reported after the second cycle was: G3 diarrhoea, G1 peripheral neuropathy, G1 Hand-Foot Syndrome, G3 thrombocytopenia and persisting G2 anaemia [3]. Chemotherapy was held on, waiting for resolution of gastrointestinal and haematological side effects.

Five weeks after the administration of the second cycle of chemotherapy, the patient presented acute painful bluish discoloration of the distal phalanxes of the fingers of the right hand. At physical examination, the right hand's fingers (excluded the thumb) were cyanotic and painful from the distal interphalangeal joints to the tips; the fingers' temperature was normal; all peripheral pulses were palpable; there were no other sites of superficial ischemia and there was no evidence of skin rashes or vasculitis. The patient confirmed that there was no family or personal history of Raynaud's phenomenon or vascular or connective tissue disease. Coagulative indexes were within normal ranges, arterial and venous doppler ultrasonography and capillaroscopy did not reveal thrombosis. In accordance with the vascular surgeon, epoetin-beta and letrozole were suspended (due to their potential property to increase haematic viscosity and thrombophilia) and fondaparinux sodium (2.5 ml/days) and polyglyan sulphate cream were administered for ten days. Specific haemostatic function tests were performed, to exclude inherited or acquired hypercoagulative state: ANA (antinuclear antibodies), ACA (anticardiolipin antibodies), APA (antiphospholipid antibodies), LAC (lupus anticoagulant), antithrombin III, protein C, protein S, Homocysteine, molecular analysis of Factor $\mathrm{V}$ Leiden and Prothrombin. All values resulted within normal ranges.

Signs and symptoms rapidly improved and completely disappeared after 7 days.

In June 2010, after 63 days from the second cycle of XELOX, the patient was admitted to hospital; blood count, liver and kidney function were normal, thus she received a third cycle of chemotherapy with FOLFOX-4 (Oxaliplatin $85 \mathrm{mg} / \mathrm{m}^{2}$ ev day 1, Folinic Acid $200 \mathrm{mg} / \mathrm{m}^{2}$ ev day 1 - 2, 5FU $400 \mathrm{mg} / \mathrm{m}^{2}$ bolus ev day 1 - 2, infusion of $5 F U 600 \mathrm{mg} / \mathrm{m}^{2}$ ev for 22 hours day 1 - 2) regimen.

Letrozole was resumed on July $1^{\text {st }} 2010$. Epoetin-beta was stopped as haemoglobin maintained $>10 \mathrm{gr} / \mathrm{dl}$.

Up to the end of December 2010, she received eight more cycles of chemotherapy with FOLFOX-4 regimen and no further sign of digital ischemia was noted.

\section{Discussion}

A review of the literature disclosed 68 previously reported cases of malignant disease associated with ischemia of the digits (Table 1). Of the total 69 cases (including the current one), the female:male ratio is about

Table 1. Published cases of malignant disease associated with digital ischemia.

\begin{tabular}{|c|c|c|c|}
\hline Type of tumor & Total (N) & $\%$ & References \\
\hline Breast & 4 & 5.9 & 4,5 \\
\hline Gastrointestinal & 13 & 19.2 & $4,5,8,13$ \\
\hline Colon & 2 & 2.9 & \\
\hline Esophagus & 3 & 4.4 & \\
\hline Pancreas & 2 & 2.9 & \\
\hline Stomach & 5 & 7.3 & \\
\hline Ileum & 1 & 1.5 & \\
\hline Gynaecologic & 9 & 13.2 & $5,7,8,11$ \\
\hline Ovary & 6 & 8.8 & \\
\hline Cervix & 2 & 2.9 & \\
\hline Uterus & 1 & 1.5 & \\
\hline Hematologic & 10 & 14.7 & $4,5,8,9$ \\
\hline Leukemia & 4 & 5.9 & \\
\hline Lymphoma & 3 & 4.4 & \\
\hline Myeloma & 3 & 4.4 & \\
\hline Lung & 10 & 14.7 & $1,5,6,12$ \\
\hline Urologic & 7 & 10.3 & $4,5,8,14$ \\
\hline Kidney & 3 & 4.4 & \\
\hline Prostate & 2 & 2.9 & \\
\hline Bladder & 1 & 1.5 & \\
\hline Testicle & 1 & 1.5 & \\
\hline Others & 10 & 14.7 & $5,8,10$ \\
\hline Head \& Neck & 2 & 2.9 & \\
\hline Atrial Mixoma & 1 & 1.5 & \\
\hline Mediastinum & 1 & 1.5 & \\
\hline Melanoma & 1 & 1.5 & \\
\hline Pheochromocytoma & 3 & 4.4 & \\
\hline Reticulum Sarcoma & 1 & 1.5 & \\
\hline Spinal cord sarcoma & 1 & 1.5 & \\
\hline Unknown primary & 5 & 7.3 & 5 \\
\hline Total & 68 & 100 & \\
\hline
\end{tabular}


4:1, which suggests that hormonal factors might be involved. The most common malignancy is gastrointestinal cancer, followed by lung, haematologic and gynaecologic malignancies. In 1987, Taylor et al. performed a prospective assessment of 700 patients who presented to their unit with finger ischemia over a 15 -year period. Only five patients had a neoplasm, that was breast carcinoma (2), chronic lymphocytic leukaemia (1), renal adenocarcinoma (1) and gastric carcinoma (1). A careful diagnostic evaluation of these cases identified three possible mechanisms underlying digital ischemia: arteritis, hyperviscosity and hypercoagulability. The author also reviewed the literature, finding 23 published case reports linking digital ischemia with malignancy: 16 cases were female and nine had gastrointestinal tumours, mostly colon, stomach, and pancreatic cancer [4].

In 1996 Shin-Fen Chow et al. described a case of ovarian cancer and gangrene of the digits and performed a review of the literature with disclosure of 57 cases of malignant disease associated with ischemia of the digits, two of which were associated with ovarian carcinoma. Approximately two-third of the patients had no predisposing factor for digital ischemia. Because of several patients had positive antinuclear antibodies, the authors proposed a vasculitis as cause of the digital ischemia. Vascular thrombosis was found in five patients. Vasoconstrictions from excessive catecholamines was thought to be responsible for three cases of pheochromocytoma. Leukocytoclastic vasculitis was found in six cases and the postulated cause was an inflammatory response in the vasculature versus immune-complexes formed by tumour- associated antigens [5].

Summing up, various mechanisms have been postulated to be the possible cause of digital necrosis: vasospasm due to sympathetic hyperactivity caused by tumour invasion or metastasis to the cervical sympathetic trunk [4-7,13]; arteritis induced by tumour antigen-antibody complexes deposition and subsequent complement activation in contact with arterial wall $[4,7,9,10,13,14]$ or as consequence of immune deregulation induced by carcinoma [5,7]; blood hyperviscosity caused by numerous factors including hypergammaglobulinemia or cryoglobulinemia [4-8,10,13,14]; hypercoagulability due to tumour products activating the haemostatic cascade and peripheral thrombosis [4-8,10,13,14].

In the case we are reporting, no definite sign of thrombosis or alteration of the coagulative system were identified. We could exclude autoimmune arteritis, thanks to the absence of antinuclear antibodiies, anticardiolipin antibodies, antiphospholipid antibody and lupus anticoagulant. Arteritis induced by tumour antigen-antibody complexes deposition in the arterial wall, microemboli and thrombosis of the microcircle have been excluded by negativity of capillaroscopy. In the same way we could exclude a thrombosis of superior upper limb thanks to arterial and venous Doppler ultrasonography.

Coagulative indexes and molecular analysis of Factor V Leiden and Prothrombin gave us the possibility to exclude an inherited or acquired hypercoagulable state. We could exclude a situation of hyperviscosity due to hypergammaglobulinemia thanks to normality of protein electrophoresis, but we could not exclude a hypervoscosity state and thrombophilia induced by epoietin-beta or letrozole.

Thrombotic events induced by letrozole account for $0.2 \%-2.8 \%$ and aromatase inhibitors (anastrazole, letrozole, examestane) are associated with lower risk of thrombotic events when compared with tamoxifen [15, 16]. Moreover, it is known that aromatase inhibitors can make manifest rheumatologic disorders, that can arise with peripheral acrocianosi.

The overall relative risk relative for a venous thromboembolic event in patient treated with the erythropoiesis-stimulating agents is 1.67 representing a $67 \%$ higher risk than placebo [17].

Venus thromboembolic events are described as rare adverse reactions to capecitabine. Moreover, it is known that the capecitabine cardiotoxicity is frequently due to coronary vasospasm [18]. Can we postulate a vasospastic activity of capecitabine on peripheral microcirculation? Of note, capecitabine has been stopped after the episode of digital ischemia, as chemotherapy was continued with FOLFOX4 regimen.

Finally, peripheral neuropathy is a characteristic toxicity related to oxaliplatin administration, that occurs in $15 \%-60 \%$ of cases [19]. Its main manifestations are finger paresthesia, numbness, tingling and cramping of the hands or feet often triggered by cold. Thus, we can not completely exclude a sympathetic neurotoxic activity due to oxaliplatin leading to peripheral vasospasm. However, if this hypothesis is reliable, we would expect a new episode of painful cyanosis when continuing with oxaliplatin administration.

In conclusion, although symptoms disappeared after few days of treatment with fondaparinux sodium, no clear thrombosis or hypercoagulative state could be demonstrated; persistent vasospasm remains the most probable cause of the digital ischemia occurred to our patient.

\section{REFERENCES}

[1] M. Konstantinos, B. Nikolaos, M. Dimitris and A. Vasilios, "Digital Ischemia as a Paraneoplastic Manifestation of Lung Cancer,” Internal Medicine, Vol. 49, No. 2, 2010, pp. 199-200. doi:10.2169/internalmedicine.49.2756 
[2] V. Ajit, “Trousseau's Syndrome: Multiple Definitions and Multiple Mechanisms,” Blood, Vol. 16, No. 6 (Supplement), 2007, pp. 1723-1729.

[3] Common Terminology Criteria for Adverse Events, Version 3.0, 2006. http://ctep.cancer.gov/protocolDevelopment/electronic_ap plications/docs/ctcaev3.pdf

[4] L. M. Taylor, M. D. Hauty, J. M. James and J. M. Edwards, "Digital Ischemia as a Manifestation of Malignancy," Annals of Surgery, Vol. 206, No. 1, 1987, pp. 62-68. doi:10.1097/00000658-198707000-00010

[5] S.-F. Chow and C. H. McKenna, "Ovarian Cancer and Gangrene of the Digits: Case Report and Review of the Literature,” Mayo Clinic Proceedings, Vol. 71, No. 3, 1996, pp. 253-258. doi:10.4065/71.3.253

[6] J. Field and I. F. Lane, "Carcinoma of the Lung Presenting with Digital Ischaemia,” Thorax, Vol. 41, No. 7, 1986, pp. 573-574. doi:10.1136/thx.41.7.573

[7] P. D. L. Maurice, "Ovarian Carcinoma and Digital Ischemia,” Clinical and Experimental Dermatology, Vol. 21, No. 5, 1996, pp. 381-382. doi:10.1111/j.1365-2230.1996.tb00130.x

[8] P. R. Hawley, A. W. Johnston and J. T. Rankin, "Association between Digital Ischemia and Malignant Disease," British Medical Journal, Vol. 3, No. 5559, 1967, pp. 208-212. doi:10.1136/bmj.3.5559.208

[9] P. A. Courtney, S. Sandhu, P. V. Gardiner and A. L. Bell, "Resolution of Digital Necrosis Following Treatment of Multiple Myeloma,” Rheumatology, Vol. 39, No. 10, 2000, pp. 1163-1164. doi:10.1093/rheumatology/39.10.1163

[10] J. R. Wright and S. Gudelis, "Digital Necrosis Associated with Squamous Cell Carcinoma of the Tonsil," Head \& Neck, Vol. 24, No. 11, 2002, pp. 1019-1021. doi:10.1002/hed.10155

[11] G. Wendela, J. P. Baars, P. C. de Jong and A. M. Westermann, "Digital Ulceration as a Paraneoplastic Syndrome in Ovarian Cancer,” Journal Of Clinical Oncology, Vol. 21,
No. 13 , 2003, pp. 2620-2625. doi:10.1200/JCO.2003.07.099

[12] P. Kopterides, T. Nikolas, T. Athanasios, P. dimitrios and L. Andreas, "Digital Gangrene and Raynaud's Phenomenon as Complications of Lung Adenocarcinoma," Lancet Oncology, Vol. 5, No. 9, 2004, p. 549. doi:10.1016/S1470-2045(04)01566-9

[13] H. Srisha and T. Gareth, "Digital Ischemia Associated with Squamous Cell Carcinoma of the Esophagus,” Digestive Disease and Science, Vol. 50, No. 4, 2005, pp. 691-693. doi:10.1007/s10620-005-2558-0

[14] M. V. Paolini, G. Jankilevinch, C. Graziano and D. S. Fernandez Romero, "Fulminant Digital Necrosis in a Patient with Prostate Adenocarcinoma," Allergology and Immunophatology (Madrid), Vol. 38, No. 1, 2010, pp. 48-50. doi:10.1016/j.aller.2009.06.006

[15] R. Ponzone, P. Minianni, E. Cassina, F. Pastorino and P. Sismondi, "Aromatase Inhibitors for Breast Cancer: Different Structures, Same Effect?” Endocrine-Related Cancer, Vol. 15, No. 1, 2008, pp. 27-36. doi:10.1677/ERC-07-0249

[16] J.-M. A. Nabholtz, "Long Term Safety of Aromatase Inhibitors in the Treatment of Breast Cancer," Therapeutics and Clinical Risk Management, Vol. 4, No. 1, 2008, pp. 189-204.

[17] M. Dicato, "Venous Thromboembolic Events and Erythropiesis Stimulating Agents: An Update," The Oncologist, Vol. 13, No. 3 (Supplement), 2008, pp.11-15. doi:10.1634/theoncologist.13-S3-11

[18] C. Ang, M. Kornbluth, M. P. Thirlwell and R. D. Rajan, "Capecitabine-Induced Cardiotoxicity: Case Report and Review of the Literature," Current Oncology, Vol. 17, No. 1, 2010, pp. 59-63. doi:10.3747/co.v17i1.437

[19] S. R. McWhinney, R. M. Goldberg and H. L. McLeod, "Platinum Neurotoxicity and Pharmacogenetics," Molecular Cancer Therapy, Vol. 8, No. 1, 2010, pp. 10-16. doi:10.1158/1535-7163.MCT-08-0840 